\title{
Hemostatic absorbable gelatin sponge loaded with 5-fluorouracil for treatment of tumors
}

\author{
This article was published in the following Dove Press journal: \\ International Journal of Nanomedicine \\ 17 April 2013 \\ Number of times this article has been viewed
}

\author{
Wei Sun' \\ Yinghui Chen ${ }^{2}$ \\ Weien Yuan ${ }^{3}$ \\ 'School of Pharmaceutical Sciences, \\ Jilin University, Changchun, People's \\ Republic of China; ${ }^{2}$ Department \\ of Neurology, Jinshan Hospital, \\ Fudan University, Jinshan District, \\ Shanghai, People's Republic of China; \\ ${ }^{3}$ School of Pharmacy, Shanghai Jiao \\ Tong University, Shanghai, People's \\ Republic of China
}

Background: Surgical tumor resection is the main treatment for tumors however the treatment process often results in massive bleeding and tumor cell residue. The main aim of this research was to address problems such as bleeding, systemic chemotherapy side effects while enhancing quality of life, and increasing drug concentrations at the tumor site by developing a novel formulation with local long-term efficacy for treatment of tumors and to stop bleeding.

Methods: 5-Fluorouracil (5-FU) was suspended in an ethyl acetate solution of poly D,Llactide-co-glycolic acid (PLGA) and a vacuum drying method was applied. The hemostatic gelatin sponge loaded with 5-FU was prepared by absorption of the suspension. The in vitro and in vivo characteristics of the hemostatic gelatin sponge loaded with 5-FU (5-FU-HAGS) were investigated.

Results: 5-FU-HAGS (hemostatic absorbable gelatin sponge loaded with 5-fluorouracil) was successfully produced with controlled release of the content and was reproducibly suitable for local tumor treatment as an implant to stop bleeding. The encapsulation efficiency of 5-FU-HAGS was above $98 \%$. The in vitro 5 -FU release kinetic profile matched a near zero-order equation for 20 days. The in vivo 5-FU plasma concentration was at a more stable level than when 5-FU solution was administered by subcutaneous injection. Bleeding can be stopped more effectively by coating a piece of blank gelatin sponge. The survival ratio of tumor-bearing mice using a 5-FU-HAGS subcutaneous implant was higher when compared to mice given a subcutaneous injection of 5-FU solution.

Conclusion: The 5-FU-HAGS system is a potential and effective way of enhancing the survival ratio and improving the quality of life of tumor-bearing mice.

Keywords: absorbable gelatin sponge, 5-fluorouracil, sustained-release, tumor

\section{Introduction}

5-Fluorouracil (5-FU) is used as a chemotherapy drug to treat pancreatic tumors, ${ }^{1,2}$ colorectal cancer, ${ }^{3-6}$ actinic keratosis, ${ }^{7,8}$ and breast tumors. ${ }^{9-12}$ Subcutaneous or intravenous injection of the drug results in systemic chemotherapy side effects, and a sustained drug delivery system at the tumor site can enhance the antitumor effects of the drug. ${ }^{13,14}$ Research has confirmed these results in animals. ${ }^{13,14} 5$-FU has been developed into different delivery systems, including biodegradable microspheres, ${ }^{15}$ 5-FU-loaded bovine serum albumin nanospheres, ${ }^{16}$ 5-FU-sustained release biodegradable microspheres of poly(methylidene malonate 2.1.2) for the treatment of brain tumor, ${ }^{17}$ suspensions of poly(D,L-lactide-co-glycolide) 5-FU-loaded microspheres for the treatment of malignant glioma, ${ }^{18} 5$-FU-loaded poly(2-hydroxyethyl methacrylateco-acrylamide) hydrogels for in vivo drug administration, ${ }^{19}$ matrix containing 5 -FU 
spraycoated with Eudragit $^{\circledR}$ S100 (Rohm GmbH \& Co., KG, Kirschenallee D-64293 Darmstadt, Germany), ${ }^{20}$ and poly(butylcyanoacrylate) nanoparticles for topical delivery of 5-FU. ${ }^{21-29}$

However, for Phase I cancer patients surgical tumor resections with chemotherapy is still the main treatment method. When these patients receive surgical tumor resections, the patients often bleed excessively, and a few tumor cells remain internally. ${ }^{30}$ In order to stop the bleeding and to assist therapy, a novel device has been developed. ${ }^{30,31}$ However, the aim of these devices was to assist therapy for the enhancement of the cells in blood or were only for in vitro studies.

Thus, in this research, hemostatic absorbable gelatin sponge loaded with 5-fluorouracil (5-FU-HAGS) were prepared by absorption, and in vitro 5-FU release and in vivo therapy effects were studied to evaluate the efficiency of these systems for 5-FU administration.

\section{Materials and methods Materials}

Poly D,L-lactide-co-glycolic acid ([PLGA] L (lactide acid):G(glycolic acid $)=50: 50$, molecular weight $47 \mathrm{kDa})$ was from Evonik Industries AG (Rellinghauser Essen, Germany). Hemostatic absorbable gelatin sponge was obtained from Nanjing Pharmaceutical Group Co., Ltd.,(Nanjing, People's Republic of China). Dichloromethane (analytical reagent), Ethyl acetate ([EA] analytical reagent), and 5-FU were purchased from Sigma-Aldrich (St Louis, MO, USA). The hemostatic gelatin sponge and 5-FU were used as received.

\section{Animals}

Male BALB/c mice (weighing $20 \pm 1$ grams at 6-8 weeks of age) were used for in vivo antitumor evaluation. Adult male Sprague-Dawley (SD) rats (weighing $200 \pm 25$ grams) were used to measure the in vivo release of 5-FU. Animals were purchased from Shanghai Laboratory Animal Center, Chinese Academy of Sciences (Shanghai, People's Republic of China). Animals were maintained under standardized conditions at a room temperature of $22^{\circ} \mathrm{C} \pm 1^{\circ} \mathrm{C}$.

\section{Preparation of 5-FU-HAGS}

5-FU was provided as a water soluble drug and the drug was directly absorbed into the pure absorbable gelatin sponge without sustained release. PLGA was suitable for sustainedrelease of 5-FU when 5-FU was suspended in PLGA organic solvents and the drug surface was coated with PLGA. ${ }^{36-40}$ To obtain sustained-release of 5-FU, 5-FU-HAGS was made with a piece of hemostatic absorbable gelatin sponge
$(2.5 \mathrm{~cm} \times 1 \mathrm{~cm})$ which fully absorbed a suspension of $1 \mathrm{~mL}$ of $10 \%$ PLGA EA solution containing $20 \mathrm{mg}$ of 5 -FU (the particle size of the drug was $5.3 \pm 1.2 \mu \mathrm{m}$ ). The suspension was made by vortex mixing for 10 minutes and was absorbed completely by the hemostatic absorbable gelatin sponge after 5 minutes. After initial drying, the 5-FU-HAGS were refrigerated $a t-4^{\circ} \mathrm{C}$ and the EA was evaporated for 24 hours, followed by further drying in a vacuum below 0.1 millibar for 48 hours at $25^{\circ} \mathrm{C} \pm 2{ }^{\circ} \mathrm{C}$ to remove remaining EA residue. The process of rapidly evaporating EA may reduce the size of the holes in the PLGA coating, and decrease the burst of 5-FU.

\section{Preparation of 5-FU-HAGS to stop bleeding}

When the freshly prepared 5-FU-HAGS had been refrigerated at $-4^{\circ} \mathrm{C}$ and the EA had evaporated for 8 hours, the pure absorbable gelatin sponge was adhered to the surface of 5-FU-HAGS and then refrigerated at $-4^{\circ} \mathrm{C}$ and the EA evaporated for 16 hours. It was further evaporated in a vacuum below 0.1 millibar for 48 hours at room temperature to remove remaining EA residue. 5-FU (20 mg) was dissolved in $0.9 \%$ sodium chloride solution and added directly to the sponge as another control group.

\section{Optical microscopic imaging of 5-FU-HAGS}

Images of 5-FU-HAGS were taken with a light microscope (SMZ445/460, Nikon Corporation, Tokyo, Japan).

\section{Encapsulation efficiency and in vitro release profiles}

The $2.5 \mathrm{~cm} \times 0.5 \mathrm{~cm}$ hemostatic absorbable gelatin sponge was used in this study. The 5-FU was absorbed uniformly in the hemostatic absorbable gelatin sponge because it had been immersed in 5-FU and PLGA organic solvent suspensions. The amount of 5-FU absorbed into the 5-FU-HAGS was determined by the process of dissolving 5-FU-HAGS in chloroform and extracting the 5-FU in phosphate buffer (1 mM, pH 7.4). 5 -FU was quantified by ultraviolet-visible (UV-Vis) spectroscopy. ${ }^{36} 5$-FU-HAGS were incubated in vials containing $3 \mathrm{~mL}$ of phosphate-buffered saline ([PBS] $100 \mathrm{mM}, \mathrm{pH} 7.4$ ) at $37^{\circ} \mathrm{C}$ with constant shaking at the rate of $150 \mathrm{rpm}$. For 5-FU release, the release medium was replaced with fresh buffer on a scheduled date and the content of 5-FU was measured by UV/Vis spectroscopy. For each sample, the 5-FU release experiment was repeated five times and release profiles were calculated based on the average of three experiments. 


\section{5-FU-HAGS administration routes for in vivo release tests}

The 15 SD rats were kept on a 12 hour light/dark cycle. The animals were fed standard rat food and water, and divided into three groups (five rats per group). Group 1 consisted of SD rats that were subcutaneously implanted in the upper part of the back with the hemostatic absorbable gelatin sponge and were used as the negative control group. Group 2 consisted of SD rats that were subcutaneously implanted in the upper part of the back with the 5-FU-HAGS and were used as the test group (34.8 $\mathrm{mg}$ of 5-FU per $\mathrm{kg}$ of body weight). Group 3 consisted of SD rats that were subcutaneously injected with $0.5 \mathrm{~mL}$ of a water solution of 5-FU and were used as the positive control group (34.8 $\mathrm{mg}$ of 5-FU per kg of body weight). The actual amount of 5-FU-HAGS that was implanted into the animals to study in vivo release was $6.96 \pm 0.87 \mathrm{mg}$.

\section{Determination of the in vivo release of 5 -FU concentration in plasma}

After the subcutaneous implant of 5-FU-HAGS and the injection of the 5-FU solutions were performed, the rats were anaesthetized using halothane at determined times. Blood samples $(0.8 \mathrm{~mL})$ from each animal were collected into heparinized ( 75 units in $15 \mu \mathrm{l}$ water) polypropylene tubes by puncturing the eye vein at $0.1,1,2,3,4,5,7,9$, $11,13,15,17$, and 20 days after implantation. The blood samples were centrifuged at $11,000 \mathrm{~g}$ for 15 minutes in an Eppendorf 5414D centrifuge (Eppendorf AG, Hamburg, Germany) immediately after collection, to obtain plasma samples. Plasma proteins were removed by precipitation by adding $2 \mathrm{M}$ trichloroacetic acid ( $6 \mu \mathrm{L}$ of trichloroacetic acid per $100 \mu \mathrm{L}$ of plasma sample), and after centrifugation (at 11,000 $\mathrm{g}$ for 6 minutes), the plasma samples were stored at $-20^{\circ} \mathrm{C}$. 5 -FU was extracted from the plasma samples with EA according to the following method: ${ }^{41}$ $100 \mu \mathrm{L}$ of PBS $(0.5 \mathrm{M}, \mathrm{pH} 8)$ and $6 \mathrm{~mL}$ of EA were added to $500 \mu \mathrm{L}$ of plasma sample, and after vigorous shaking for 6 minutes and centrifugation ( $4000 \mathrm{~g}, 3$ minutes), the EA phase was collected. The EA phase was evaporated using nitrogen gas at $55^{\circ} \mathrm{C}$ and the dried samples were redissolved with $100 \mu \mathrm{L}$ of $0.01 \mathrm{M}$ potassium dihydrogen phosphate $\left(\mathrm{KH}_{2} \mathrm{PO}_{4}, \mathrm{pH} 4\right)$. The 5-FU concentration in the plasma samples was measured by high performance liquid chromatography (Shimadzu LC-10ATVP, Shimadzu Corporation, Kyoto, Japan). The separation column was a reversed-phase C18 column (Atlantis T3 Column, $100 \AA, 3 \mu \mathrm{m}, 1 \mathrm{~mm} \times 150$ mm, 1/pkg; Waters Corporation, Milford, MA, USA). The mobile phase was $0.01 \mathrm{M} \mathrm{KH}_{2} \mathrm{PO}_{4}(\mathrm{pH} 4)$. The flow rate of the mobile phase was $1 \mathrm{~mL} / \mathrm{min}$ and the wavelength of the detector was $266 \mathrm{~nm}$. In order to measure the content of 5-FU, 5-FU standards of $0.1-100 \mu \mathrm{g} / \mathrm{mL}$ in PBS $(1 \mathrm{mM}$, $\mathrm{pH} 7.4)$ were measured and pooled drug-free plasma with known amounts of 5-FU dissolution in plasma were used. In both cases, 5-FU standard curves were measured for external standardization, and linear curves with a correlation coefficient of 0.987 were produced from the area under the UV absorption peak measurements. The 5-FU retention time was $10.4 \pm 0.3$ minutes. From the area under the UV absorption peak measurements of plasma 5-FU concentration versus time, a decreased rate of 5-FU concentration in plasma was calculated by the slope of the straight line from the time of maximum 5-FU concentration, the absence of 5-FU in plasma, and the circulating blood volume of SD rats. For calculating the circulating blood volume of SD rats, the recommended mean value of $64 \mathrm{~mL} / \mathrm{kg}$ of body weight was used. ${ }^{32}$

\section{5-FU-HAGS administration routes for in vivo efficacy studies}

The local antitumor efficacy studies were carried out in male BALB/c mice bearing colon carcinoma. All animal experiments were performed in accordance with protocols approved by the Institutional Animal Care and Use Committee of Shanghai Jiao Tong University, Shanghai, People's Republic of China. For in vivo implantation, CT26.WT cells were suspended in PBS (pH 7.4) and injected subcutaneously in the dorsal cervical at a concentration of $5 \times 10^{6}$ cells in $0.2 \mathrm{~mL}$ of PBS. When the tumor volume reached the expected size, the mice were randomly divided into three groups $(\mathrm{n}=10)$, and that day was defined as day 0 . Group 1 consisted of BALB/c mice that were subcutaneously implanted with the hemostatic absorbable gelatin sponge at the tumor site and were the negative control group. Group 2 consisted of BALB/c mice that were subcutaneously implanted with the 5-FU-HAGS at the tumor site and were the study group (34.8 $\mathrm{mg}$ of 5-FU per kg of weight). Group 3 consisted of BALB/c mice that were subcutaneously injected with $0.2 \mathrm{~mL}$ of $5-\mathrm{FU}$ in water at the tumor site and were the positive control group $(34.8 \mathrm{mg} / \mathrm{kg} 5$-FU solution was administrated once). All the mice were numbered, and the tumor length and width were measured before each administration. The tumor volume was calculated according to the formula: volume $=0.5 \times\left(\mathrm{L} \times \mathrm{W}^{2}\right)$, where $\mathrm{L}$ is the tumor major axis. Survival rate of effect was studied according to 


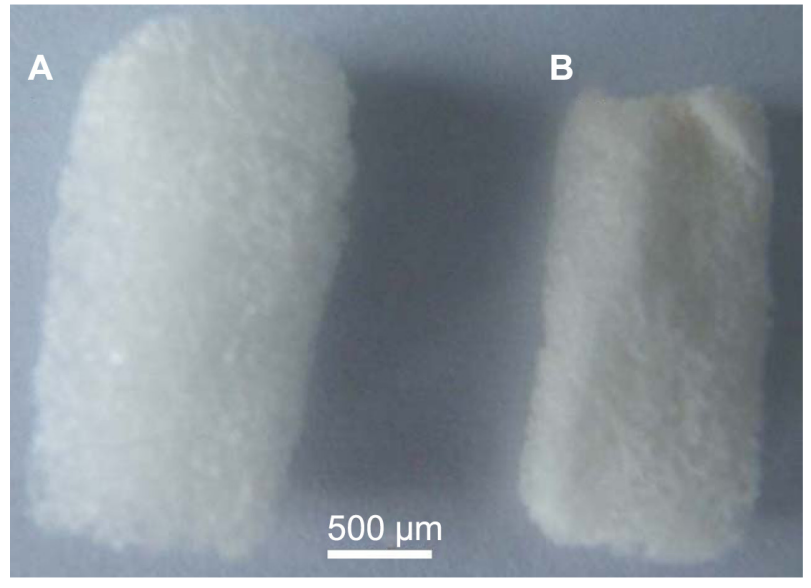

Figure I Microscope images of 5-fluorouracil-loaded hemostatic gelatin sponges. (A) Blank hemostatic gelatin sponge and (B) 5-fluorouracil-loaded hemostatic gelatin sponge.

the Survival rate of effect $=\mathrm{S} \times 100 \% / \mathrm{T}$, (S: survival mice, $\mathrm{T}$ :sum test mice) of the number of therapy mice.

\section{The effect on tumor recurrence after resection with 5-FU-HAGS}

In order to study the effect on tumor recurrence after resection with 5-FU-HAGS, 20 male BALB/c colon carcinoma-bearing mice were randomly divided into two groups, group 1 and group 2, when the average volume of the tumor was $400 \pm 45 \mathrm{~mm}^{3}$. The 5-FU-HAGS was used to stop bleeding and for local therapy after resection of the tumors in group 1. Gelatin sponge alone was used to stop bleeding and was the control in group 2. The volume of tumor was $200 \mathrm{~mm}^{3}$ and this was the standard for tumorigenesis.

\section{Statistical analysis}

The data were expressed as the mean \pm standard deviation. Statistically significant differences were determined using the one-way ANOVA test and $P<0.05$ was considered to be statistically significant.

\section{Results and discussion Morphology of the 5-FU-HAGS}

The morphology of the 5-FU-HAGS was characterized with an Olympus $\mu 710$ camera (Olympus Corporation, Tokyo, Japan). Figure 1 shows the morphology of the 5-FU-HAGS and HAGS. We found that the 5-FU-HAGS were smaller in volume than free 5-FU-HAGS. This was possibly because the hemostatic absorbable gelatin sponge had adsorbed the 5-FU PLGA suspension and the PLGA had made the gelatin sponge compressed and smaller in volume.

\section{Encapsulation efficiency and release kinetics for 5-FU-HAGS}

The encapsulation efficiency of 5-FU-HAGS was $95.5 \% \pm 3.9 \%$, and the encapsulation efficiency of 5-FU alone absorbed into the hemostatic absorbable gelatin sponge was $40.5 \% \pm 4.9 \%$. This was because 5 -FU powder alone precipitated in the EA solution, while the 5-FU powder suspended in PLGA EA solution deposited less in comparison. The PLGA EA solution was more viscous than the EA solution.

Figure 2 summarizes the 5-FU release profile for 5-FUHAGS. The 5-FU-HAGS showed an almost zero-order 5-FU release profile (the release kinetics equation was: $\mathrm{Q}_{\mathrm{A}}=3.1822 \mathrm{t}+13.774, \mathrm{R}^{2}=0.9938$, (where $\mathrm{QA}=$ amount of release $5-\mathrm{FU} ; \mathrm{t}=$ release time) from day 1 to day 25$)$. This was possibly because the degradation rate of the hemostatic

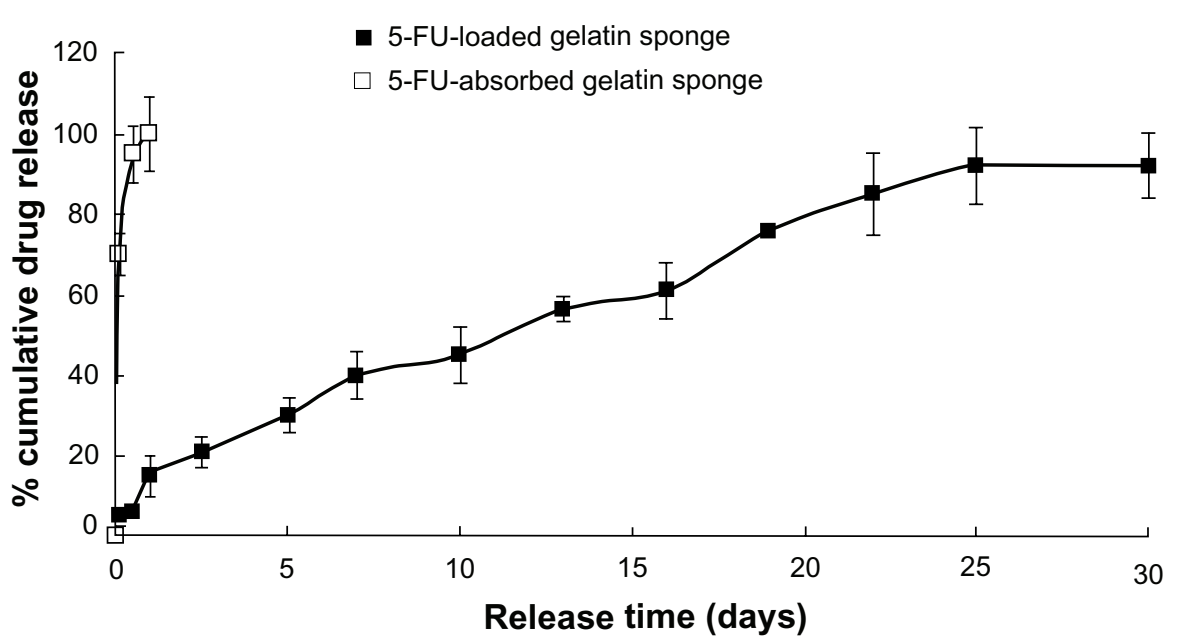

Figure 2 In vitro release profile of the 5 -fluorouracil-loaded hemostatic gelatin sponge $(n=5)$. Abbreviation: 5-FU, 5-fluorouracil. 
absorbable gelatin sponge was more rapid than the PLGA degradation rate when the 5-FU-HAGS was incubated in $2 \mathrm{~mL}$ of PBS at $37^{\circ} \mathrm{C} . .^{30,31}$ The degradation of this hemostatic absorbable gelatin sponge led to sufficient penetration of water and produced drug molecular diffusion channels for 5-FU release. At the same time, the 5-FU diffusion distance and concentration from inner to outer of the sponge decreased due to PLGA and hemostatic absorbable gelatin sponge degradation, so the release of 5-FU remained at a relatively constant rate (namely by a combination 5-FU release course of erosion and diffusion). The result that we reported in previous studies were observed. ${ }^{30,31}$ The release profile of 5-FU alone absorbed in the hemostatic absorbable gelatin sponge was almost $100 \%$ in 2 hours. This was because the
PLGA had sustained-release for 5-FU when it was suspended in PLGA organic solvents and the drug surface was coated with PLGA. Because 5-FU was water soluble, the drug was directly absorbed into the pure absorbable gelatin sponge without sustained-release.

\section{Release of 5-FU in the in vivo rat study}

The release of 5-FU from the 5-FU-HAGS concentration of 5-FU in plasma with time (Figure 3). There was a rapid release of 5-FU from the 5-FU-HAGS during the initial release phase, and the 5-FU concentration in plasma reached a maximum (Figure $3 \mathrm{~A}$ ) of $2.3 \pm 0.23 \mu \mathrm{g} / \mathrm{mL}$ from 5 -FUHAGS at 24 hours after implant, and relatively more stable plasma concentrations were sustained from day 2 to day 20
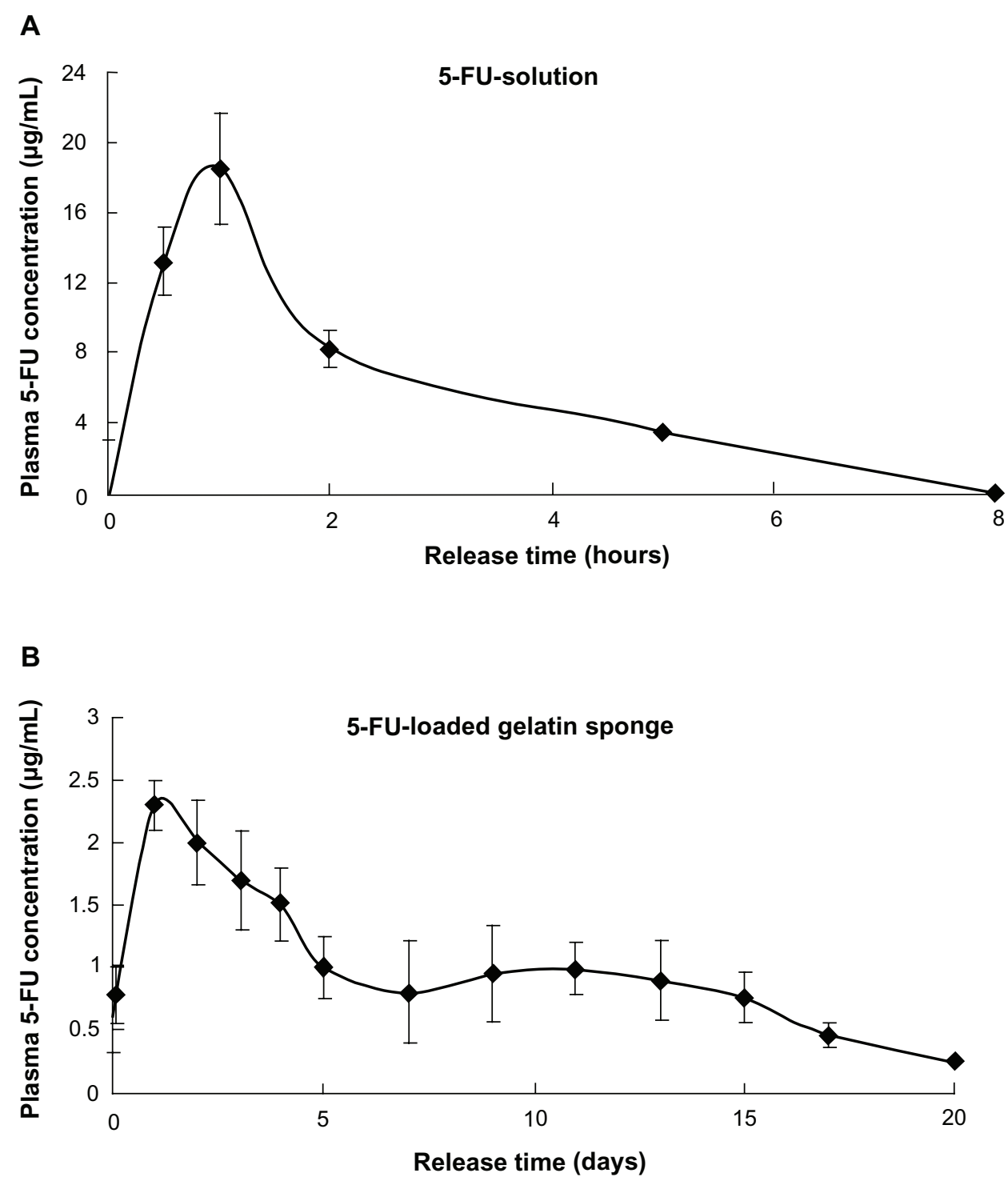

Figure 3 In vivo plasma concentration of 5-fluorouracil-loaded hemostatic gelatin sponge (administration dose: $34.8 \mathrm{mg} / \mathrm{kg}$ once; $\mathrm{n}=5$ ). (A) 5 -Fluorouracil (5-FU) solution and (B) 5-FU-loaded hemostatic absorbable gelatin sponge.

Abbreviation: 5-FU, 5-fluorouracil. 


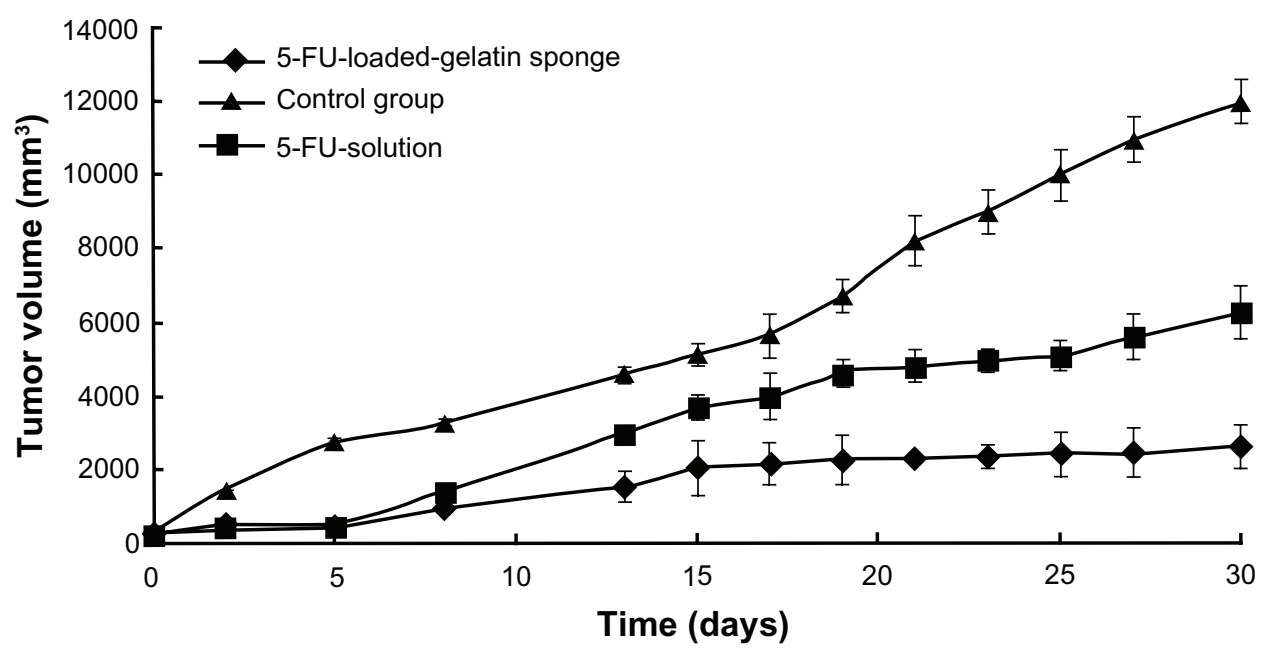

Figure 4 Effect of 5 -fluorouracil-loaded hemostatic gelatin sponge on tumor volume.

Notes: Error bars represent the standard deviation for 10 mice per group. $P<0.05$ calculated using a one-way ANOVA test. Abbreviation: 5-FU, 5-fluorouracil.

when compared to the subcutaneous 5-FU solutions that were injected. A decrease in the rate of 5-FU concentration in plasma could be calculated from the straight line slope from the time of maximum 5-FU concentration in plasma to the time of free drug in plasma, and the circulating blood volume of rats. The circulating blood volume of rats $^{32}$ is $58-70 \mathrm{~mL} / \mathrm{kg}$ of body weight, and the suggested mean value was $64 \mathrm{~mL} / \mathrm{kg}$ of body weight. ${ }^{32}$ Thus, the decrease in the rate of 5 -FU concentration in plasma was $2.4 \pm 0.18 \mu \mathrm{g} /$ day. Subcutaneous administration of 5-FU water solutions at $34.8 \mathrm{mg} / \mathrm{kg}$ doses resulted in a maximum 5 -FU concentration of $17.9 \pm 1.1 \mu \mathrm{g} / \mathrm{mL} 30$ minutes after administration, and 5-FU was measured in plasma for 5 hours (Figure 3B). The area under the plasma level versus time curve of 5-FU implanted from 5-FU-HAGS was 12 times more than that of subcutaneously injected 5-FU in water. This was possibly because the 5-FU-HAGS always stably released 5-FU in plasma and resulted in keeping the drug concentration in plasma over a longer of time, and could reduce the sum clearance rate of the drug in plasma.

\section{In vivo efficacy study and toxicity}

To compare the antitumor efficacy of 5-FU-HAGS and the 5-FU aqueous solution, BALB/c mice bearing colon carcinoma were either subcutaneously implanted with 5-FUHAGS at the tumor site, or subcutaneously injected with 5-FU aqueous solution at $34.8 \mathrm{mg} / \mathrm{kg}$ with $0.5 \%$ sodium chloride (continuous infusion of $34.8 \mathrm{mg} / \mathrm{kg} 5$-FU once as the control group). Changes in tumor volume, tumor weight, and the survival rate of mice were measured for 30 days. Figures 4 and 5 show that 5-FU-HAGS or 5-FU aqueous solution achieved a significant increase in antitumor efficacy and enhanced the survival rate of mice compared to that of the control group. The lower ratio of tumor volume to weight, and the higher survival ratio from the 5-FU-HAGS group surpassed that of the group administered with the 5-FU aqueous solution. The toxicity of 5-FU-HAGS was evaluated by detecting changes in body weight. The change in body weight of mice in the control group, the group administered with 5-FU solution, and the group given 5-FU-HAGS was monitored every 3 days during the experiment. However, no significant differences were observed in the body weights between the untreated groups (no 5-FU-HAGS) and the group treated with 5-FU-HAGS $(P>0.05)$. The study showed that bleeding had stopped in mice in the 5-FU-HAGS group and only decreased $20 \%$ more of the bleeding time than that of blank gelatin sponge. When the pure hemostatic gelatin sponge was attached to the 5-FU-HAGS, the bleeding had stopped and this effect was almost equal to that observed with the pure hemostatic

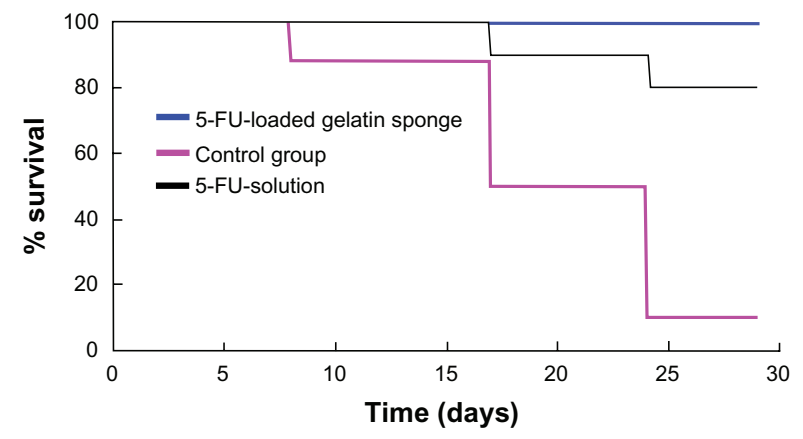

Figure 5 Effect of the 5 -fluorouracil-loaded hemostatic gelatin sponge on survival. Abbreviation: 5-FU, 5-fluorouracil. 


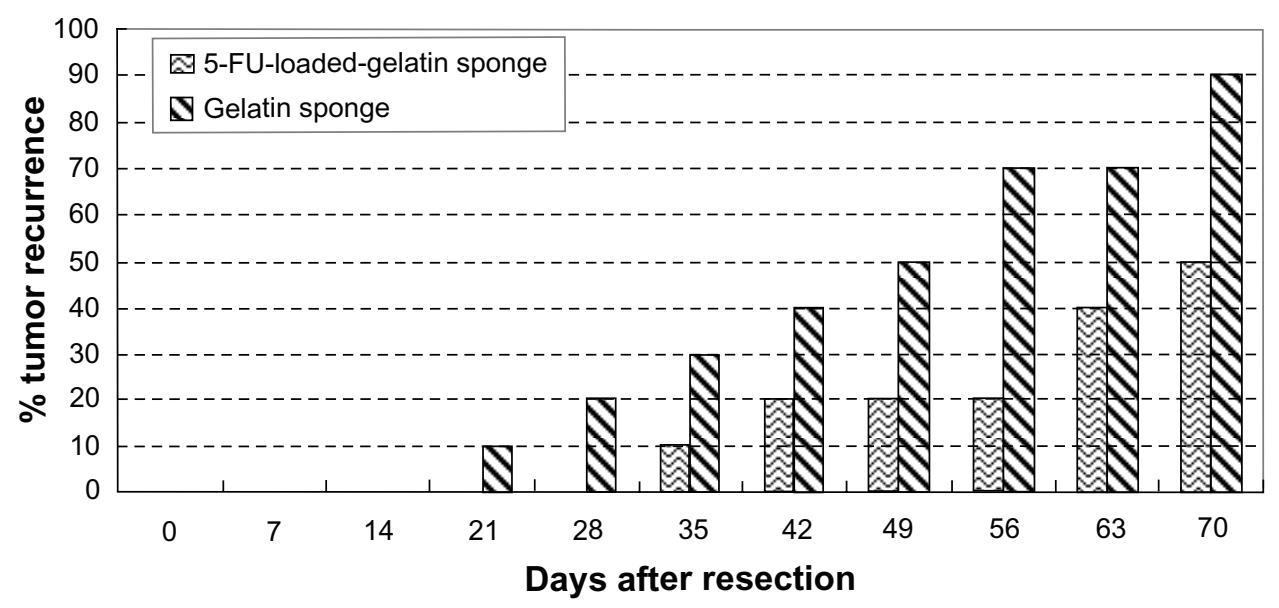

Figure 6 The effect of tumor recurrence after resection with the use of 5-fluorouracil gelatin sponge and gelatin sponge alone. Abbreviation: 5-FU, 5-fluorouracil.

gelatin sponge. These results suggest that the 5-FU-HAGS equaled to stopping bleeding work of the HAGS.

The intratumor administration of a sustained-release antitumor drug system to tumor-bearing mice ${ }^{33,34}$ showed significantly greater antitumor effect on the tumor growth inhibition ratio and on the survival period when compared to drug solutions alone. The antitumor efficacy of 5-FU-loaded microspheres was studied in tumor-bearing rats after injection of C6 malignant glioma cells in the brain, and this study showed a significant decrease in the mortality of rats treated with 5-FU-loaded microspheres when compared with the group injected with 5-FU solution..$^{35}$ The therapy effect of 5-FU-HAGS were the same as the therapy effect when the tumor remission was carried out and bleeding stopped with drug chemotherapy. The 5-FU-HAGS had sustained-release of 5-FU and the hemostatic absorbable gelatin sponge also stopped bleeding. The study showed a decrease in tumor recurrence after resection with the use of 5-FU-HAGS when compared to gelatin sponge alone (Figure 6).

\section{Conclusion}

5-FU-HAGS was developed for sustained-release of 5-FU, local tumor targeting, and to stop bleeding. 5-FU-HAGS were manufactured with biocompatible and biodegradable PLGA and gelatin sponge materials. According to our experimental data, sustained-release of 5-FU from the 5-FU-HAGS occurred for approximately 20 days in vitro and in vivo. In addition, the antitumor effects were also studied in tumor-bearing BALB/c mice. These antitumor effects were significantly enhanced by implanting 5-FU-HAGS in mice rather than by injecting mice with 5 -FU in aqueous solution. Local administration of 5-FU-HAGS improved the drug concentration accumulation in tumor tissues; therefore, 5-FU-HAGS may enhance antitumor effects in tumors and decrease drug toxicity in normal tissues. As a result, implantable 5-FU-HAGS delivery systems may be used to stop bleeding and as a good local therapy method for tumor targeting of chemotherapy agents after tumor resection for solid tumors.

\section{Acknowledgment}

We appreciate the generous help of the Instrumental Analysis Centre of Shanghai Jiao Tong University.

\section{Disclosure}

The authors have no conflicts of interest in this work.

\section{References}

1. Günzburg WH, Löhr M, Salmons B. Novel treatments and therapies in development for pancreatic cancer. Expert Opin Invest Drugs. 2002; 11:769-786.

2. Li D, Xie K, Wolff R, Abbruzzese JL. Pancreatic cancer. Lancet. 2004;363:1049-1057

3. Moehler M, Teufel A, Galle PR. New chemotherapeutic strategies in colorectal cancer. Recent Results Cancer Res. 2005;165:250-259.

4. Segura C, Afchain P, de Gramont A, André T. Adjuvant treatment of colorectal cancer. Bull Du Cancer. 2006;93:683-690.

5. El-Khoueiry AB, Lenz HJ. Should continuous infusion 5-fluorouracil become the standard of care in the USA as it is in Europe? Cancer Invest. 2006;24:50-55.

6. Goldberg RM, Sargent DJ, Morton RF, et al. Randomized controlled trial of reduced-dose bolus fluorouracil plus leucovorin and irinotecan or infused fluorouracil plus leucovorin and oxaliplatin in patients with previously untreated metastatic colorectal cancer: a North American Intergroup Trial. J Clin Oncol. 2006;24:3347-3353.

7. Jorizzo J. Topical treatment of actinic keratosis with fluorouracil: is irritation associated with efficacy? J Drug Dermatol. 2004;3:21-25.

8. Gupta AK, Davey V, Mcphail H. Evaluation of the effectiveness of imiquimod and 5-fluorouracil for the treatment of actinic keratosis: critical review and meta-analysis of efficacy studies. J Cutan Med Surg. 2005;9:209-214. 
9. Yarde Y, Baselga J, Miles D. Molecular approach to breast cancer treatment. Semin Oncol. 2004;31:6-13.

10. Hutchins LF, Green SJ, Ravdin PM, et al. Randomized, controlled trial of cyclophosphamide, methotrexate, and fluorouracil versus cyclophosphamide, doxorubicin, and fluorouracil with and without tamoxifen for high-risk, node-negative breast cancer: treatment results of Intergroup Protocol INT-0102. J Clin Oncol. 2005;23:8313-8321.

11. Eniu A, Carlson RW, Aziz Z, et al. Breast cancer in limited-resource countries: treatment and allocation of resources. Breast J. 2006;12: S38-S53.

12. Roüessé J, de la Lande, B, Bertheault-Cvitkovic F, et al. A phase III randomized trial comparing adjuvant concomitant chemoradiotherapy versus standard adjuvant chemotherapy followed by radiotherapy in operable node-positive breast cancer: final results. Int J Radiat Oncol Biol Phys. 2006;64:1072-1080.

13. Roullin VG, Mege M, Lemaire L, et al. Influence of 5-fluorouracil-loaded microsphere formulation on efficient rat glioma radiosensitization. Pharm Res. 2004;21:1558-1563.

14. Larina IV, Evers BM, Ashitkov TV, et al. Enhancement of drug delivery in tumors by using interaction of nanoparticles with ultrasound radiation. Technol Cancer Res Treat. 2005;4:217-226.

15. Muvaffak A, Gurhan I, Gunduz U, Hasirci N. Preparation and characterization of a biodegradable drug targeting system for anticancer drug delivery: microsphere-antibody conjugate. J Drug Target. 2005;13: 151-159.

16. Santhi K, Dhanaraj SA, Vinod J, et al. A study on the preparation and anti-tumor efficacy of bovine serum albumin nanospheres containing 5-fluorouracil. Drug Dev Ind Pharm. 2002;28:1171-1179.

17. Fournier E, Passirani C, Vonarbourg A, et al. Therapeutic efficacy of novel 5-FU-loaded PMM 2.1.2-based microspheres on C6 glioma. Int J Pharm. 2003;268:31-35.

18. Menei P, Jadaud E, Faisant N, et al. Stereotaxic implantation of 5-fluorouracil releasing microspheres in malignant glioma. A phase I study. Cancer. 2004;100:405-410.

19. Blanco MD, Garcia O, Gomez C, et al. In-vivo drug delivery of 5-fluorouracil using poly(2-hydroxyethyl methacrylate-coacrylamide) hydrogels. J Pharm Pharmacol. 2000;52:1319-1325.

20. Zambito Y, Baggiani A, Carelli V, et al. Matrices for site-specific controlled-delivery of 5-fluorouracil to descending colon. J Control Release. 2005;102:669-677.

21. Simeonova M, Velichkova R, Ivanova G, et al. Poly(butylcyanoacrylate) nanoparticles for topical delivery of 5-fluorouracil. Int J Pharm. 2003;263:133-140.

22. Nair KL, Jagadeeshan S, Nair SA, Kumar GS. Biological evaluation of 5-fluorouracil nanoparticles for cancer chemotherapy and its dependence on the carrier, PLGA. Int J Nanomedicine. 2011;6:1685-1697.

23. Ma J, Chen CS, Blute T, Waxman DJ. Anti-angiogenesis enhances intratumoral drug retention. Cancer Res. 2011;71:2675-2685.

24. Banerjee S, Sen K, Pal TK, Guha SK. Poly(styrene-co-maleic acid)based $\mathrm{pH}$-sensitive liposomes mediate cytosolic delivery of drugs for enhanced cancer chemotherapy. Int J Pharm. 2012;436:786-797.

25. Ragab DM, Rohani S, Consta S. Controlled release of 5-fluorouracil and progesterone from magnetic nanoaggregates. Int J Nanomedicine. 2012;7:3167-3189.
26. Du JZ, Du XJ, Mao CQ, Wang J. Tailor-made dual pH-sensitive polymerdoxorubicin nanoparticles for efficient anticancer drug delivery. $\mathrm{J} \mathrm{Am}$ Chem Soc. 2011;133:17560-17563.

27. Ren X, Zheng N, Gao Y, Chen T, Lu W. Biodegradable three-dimension micro-device delivering 5-fluorouracil in tumor bearing mice. Drug Deliv. 2012;19:36-44.

28. Choi KY, Yoon HY, Kim JH, et al. Smart nanocarrier based on PEGylated hyaluronic acid for cancer therapy. ACS Nano. 2011;5:8591-8599.

29. Li J, Hou N, Faried A, Tsutsumi S, Kuwano H. Inhibition of autophagy augments 5-fluorouracil chemotherapy in human colon cancer in vitro and in vivo model. Eur J Cancer. 2010;46:1900-1909.

30. Yuan W, Liu Z. Surgical wound healing using hemostatic gauze scaffold loaded with nanoparticles containing sustained-release granulocyte colony-stimulating factor. Int J Nanomedicine. 2011;6:3139-3149.

31. Xu L, Wu F, Yuan W, Jin T. Controlled-release implant system formulated using biodegradable hemostatic gauze as scaffold. Int J Pharm. 2008;355:249-258.

32. Diehl KH, Hull R, Morton D, et al. A good practice guide to the administration of substances and removal of blood, including routes and volumes. J Appl Toxicol. 2001;21:15-23.

33. Konishi M, TabataY, Kariya M, et al. In vivo anti-tumor effect of dual release of cisplatin and adriamycin from biodegradable gelatin hydrogel. J Control Release. 2005;103:7-19.

34. Obara K, Ishihara M, Ozeki Y, et al. Controlled release of paclitaxel from photocrosslinked chitosan hydrogels and its subsequent effect on subcutaneous tumor growth in mice. J Control Release. 2005;110:79-89.

35. Menei P, Boisdron-Celle M, Crousé A, et al. Effect of stereotactic implantation of biodegradable 5-fluorouracil-loaded microspheres in healthy and C6 glioma-bearing rats. Neurosurgery. 1996;39:117-124.

36. Blanco MD, Sastre RL, Teijón C, Olmo R, Teijón JM. 5-Fluorouracilloaded microspheres prepared by spray-drying poly(D,L-lactide) and poly(lactide-co-glycolide) polymers: characterization and drug release. J Microencapsul. 2005;22:671-682.

37. Yuan W, Liu Z. Controlled release and preserved bioactivity of proteins from (self-assembled) core-shell double walled microspheres. Int $J$ Nanome. 2012;7:257-270.

38. Rong X, Mo X, Ren T, et al. Neuroprotective effect of erythropoietinloaded composite microspheres on retinal ganglion cells in rats. Eur $J$ Pharm Sci. 2011;43:334-342.

39. Ren T, Yuan W, Zhao H, Jin T. Sustained-release polylactide- co-glycolide microspheres loaded with pre-formulated protein polysaccharide nanoparticles. Micro \& Nano Letters, 2011;6(2):70-74.

40. Yuan W,Zhang Y, Wu F, et al. Jin T. Preparation of protein-loaded sustained-release microspheres via'solid-in-oil-in- hydrophilic oilin-ethanol (S/O/hO/E)' emulsification. Colloids Surf B, 2010; 79: 326-333.

41. Buckpitt AR, Boyd MR. A sensitive method for determination of 5-fluorouracil and 5-fluoro-2'-deoxyuridine in human plasma by highpressure liquid chromatography. Anal Biochem. 1980;106:432-437.
International Journal of Nanomedicine

\section{Publish your work in this journal}

The International Journal of Nanomedicine is an international, peerreviewed journal focusing on the application of nanotechnology in diagnostics, therapeutics, and drug delivery systems throughout the biomedical field. This journal is indexed on PubMed Central, MedLine, CAS, SciSearch $\AA$, Current Contents ${ }^{\circledR} /$ Clinical Medicine,
Dovepress

Journal Citation Reports/Science Edition, EMBase, Scopus and the Elsevier Bibliographic databases. The manuscript management system is completely online and includes a very quick and fair peer-review system, which is all easy to use. Visit http://www.dovepress.com/ testimonials.php to read real quotes from published authors. 